\title{
Stiffness and moisture content prediction model of wooden veneer using fast online near-infrared (NIR) spectroscopic system
}

\begin{abstract}
The fastest developed online near-infrared (NIR) spectroscopic system with the speed of $120 \mathrm{~m} / \mathrm{min}$ was applied to predict the quality of wooden veneer, i.e., the stiffness (modulus of elasticity/MOE) and the moisture content (MC) from two species, Japanese cedar (Cryptomeria japonica) and Douglas-fir (Pseudotsuga menziesii). The measurement of MOE was conducted by three-point bending test on air-dry MC (12-13\%) and the MC measurement was conducted from $30 \%$ to $12 \%$. The root mean square error of crossvalidation (RMSECV) values from MOE prediction for Japanese cedar veneer and Douglasfir veneer are $0.9 \mathrm{GPa}$ and $1.8 \mathrm{GPa}$, while the RMSECV values from MC prediction are $1.0 \%$ and $0.6 \%$ for Japanese cedar veneer and Douglas-fir veneer, respectively. This fast speed of online NIR spectra measurement could give better prediction accuracy and be applied in the plywood production industrial line to increase the efficiency.
\end{abstract}

Keywords: nondestructive measurement, nir spectroscopy, veneer, modulus of elasticity, moisture content
Volume I Issue 2 - 2017

\author{
Imran Arra'd Sofianto,' Tetsuya Inagaki, \\ Mariya Itoh, ${ }^{2}$ Satoru Tsuchikawa' \\ 'Department of Biosphere Resources Science, Nagoya \\ University, Japan \\ 2lida Kogyo Co Ltd, Japan
}

Correspondence: Satoru Tsuchikawa, Graduate School of Bioagricultural Sciences, Nagoya University, Furo-cho, Chikusaku, Nagoya, 464-860I, Japan, Tel +8I-52-789-4I57, Fax +8I-52789-4157, Email st3842@agr.nagoya-u.ac.jp

Received: September II, 2017 | Published: September 28, 2017
Abbreviations: NIR, near-infrared; MAFF, ministry of agriculture, forestry, and fisheries; QC, quality control; MOE, modulus of elasticity; MC, moisture content; SG, specific gravity; $\mathrm{S} / \mathrm{N}$, signal-to-noise; LOO, leave-one-out; CV-PLSR, cross-validation partial least square regression; SNV, standard normal variate; MSC, multiplicative scatter correction; SP, smoothing-point; RMSECV, root mean square error for cross-validation; LV, latent variables

\section{Introduction}

Plywood production in Japan plays an important role in providing the materials for wooden building construction. The Japanese Government's Ministry of Agriculture, Forestry, and Fisheries (MAFF) confirmed in 2015 that the share of domestic wood used for plywood production was rising, reaching approximately $70 \%$ in 2014 , although plywood was only $30 \%$ of the total wood supply. The 186 plywood mills in Japan in 2014 produced 2,18million $\mathrm{m}^{3} /$ year of general plywood. ${ }^{1}$ Novel technology to increase the productivity on the plywood production line is sorely required.

Near-infrared (NIR) spectroscopy, which has been being widely used in the field of wood science in the last 20 years, seems helpful for increasing the efficiency in the quality control (QC) of the product. NIR spectroscopy, as advanced non-destructive testing technology, shows promising application, as transmitted or reflected spectra of electromagnetic waves ranging from 800 to $2500 \mathrm{~nm}$ have been used for the online or on-site measurement of various organic materials, such as agricultural products, foods, polymers, textiles, pharmaceuticals, and petrochemicals. ${ }^{2}$ Newly developed technology using NIR spectroscopy should be useful to quickly assess the quality of the wooden veneer, i.e., the stiffness (modulus of elasticity/MOE) and the moisture content (MC), without destroying the materials.

Studies on veneer using NIR technology have been done by many researchers. Dry veneer stiffness was predicted using NIR spectra by Fujimoto et al. ${ }^{3}$ from transverse sections of the logs. Prediction of Yellow poplar (Liriodendron tulipifera) veneer stiffness and bulk density using NIR and multivariate calibration was done by Adedipe et al. ${ }^{4}$ Koumbi-Mounanga et al. ${ }^{5}$ Examined the surface quality sensing (MC, surface energy characteristics, and adhesive bond strength) of Trembling aspen (Populus tremuloides Michx.) veneers products by NIR spectroscopy. Carneiro et al. ${ }^{6}$ Stated that assessment of the flexural static MOE and specific gravity (SG) of veneers is important for quality control and cited it as their reason doing research on NIR and chemo metrics for predicting SG and flexural MOE of Pinus spp. Veneers. Meder et al. ${ }^{7}$ developed an online NIR spectra measurement system with the speed of $60 \mathrm{~m} / \mathrm{s}$ to predict veneer stiffness from miniLVL test panels.

This research attempts to use modeling to predict MOE and MC of wooden veneer (Japanese cedar and Douglas-fir) for a real sample using fast developed online NIR spectroscopic system, whose conveyor run at $120 \mathrm{~m} / \mathrm{min}$, taking into consideration future wood industry application. The prediction model resulting from this research is compared to the previous research.

\section{Materials and method}

\section{Materials}

In this research, 216 veneers of Japanese cedar (Cryptomeria japonica) with the dimension size of $960 \mathrm{~mm}(\mathrm{~L}) \times 300 \mathrm{~mm}(\mathrm{~T})$ $\mathrm{x} 3.1 \mathrm{~mm}(\mathrm{R})$ and 160 veneers of Douglas-fir (Pseudotsuga menziesii) with the dimension size of $1000 \mathrm{~mm}(\mathrm{~L}) \times 300 \mathrm{~mm}(\mathrm{~T}) \times 3.2 \mathrm{~mm}(\mathrm{R})$ were used for MOE measurement and 10 others veneers of each species were used for MC measurement. All veneers samples were purchased from the plywood factory in Nakatsugawa, Gifu, Japan. All veneers samples were manufactured by rotary cut resulting on tangential surface of the veneer as shown in (Figure 1). 

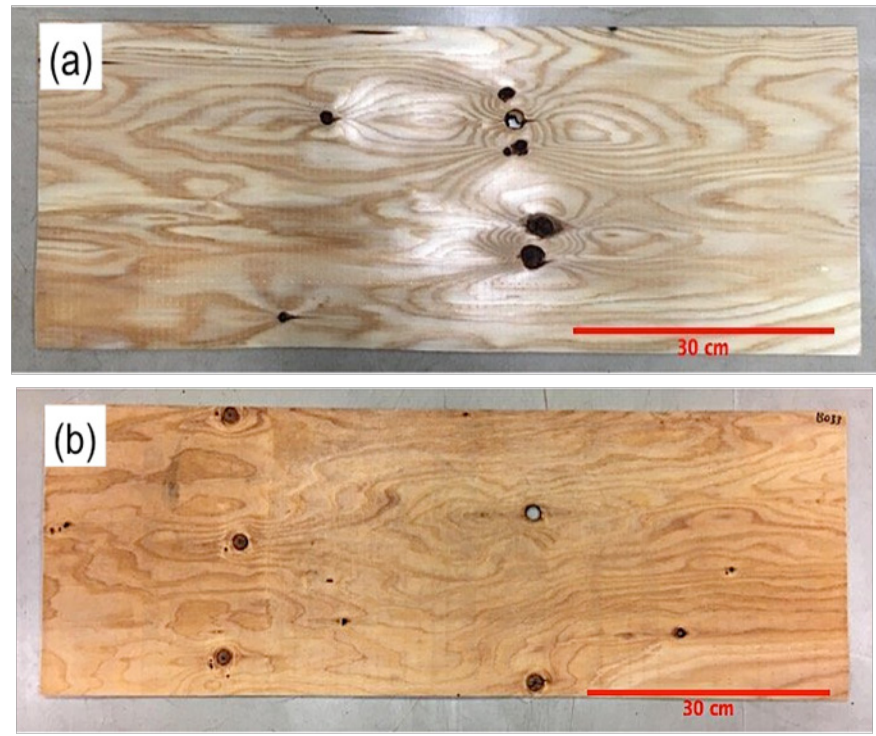

Figure I (A) Japanese cedar veneer and (B) Douglas-fir veneer.

\section{Methods}

The gigapascal (GPa) measurement of MOE was conducted by three-point bending test on air-dry $\mathrm{MC}$ condition (12-13\%) as shown in Figure 2 and the MC measurement was conducted from $30 \%$ to $12 \%$ with $68 \mathrm{MC}$ values obtained from Japanese cedar veneers and $138 \mathrm{MC}$ values obtained from Douglas-fir veneers (from both each species $10 \mathrm{MC}$ veneers samples). The measurement of online NIR spectra for MOE was conducted after three-point bending test for MOE measurement as well as the measurement of online NIR spectra for $\mathrm{MC}$ was conducted after measuring the weight of the $\mathrm{MC}$ veneer samples.

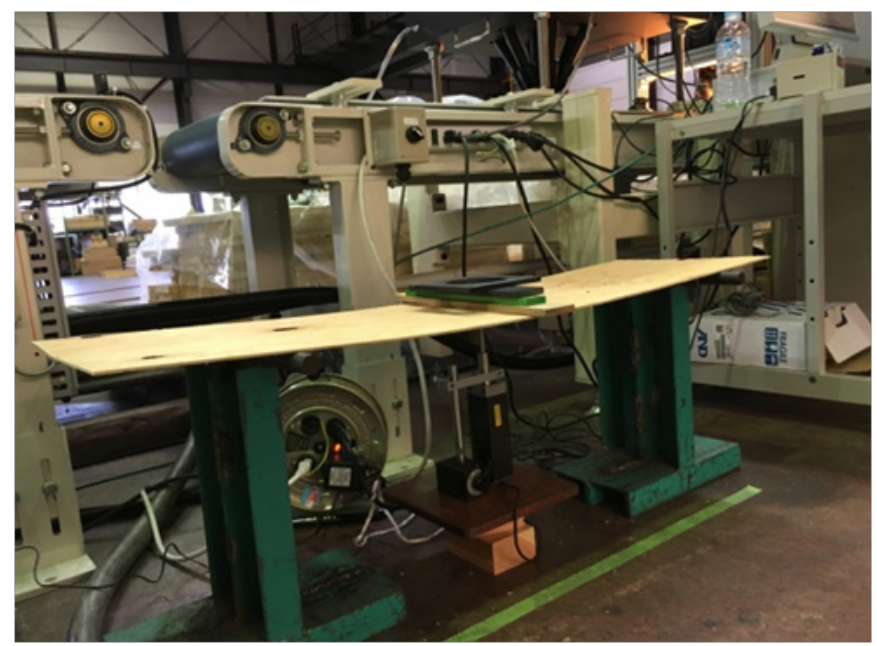

Figure 2 Three-point bending test measurement for Douglas-fir veneer.

The online NIR spectroscopic system was used for the measurements as shown in Figure 3. The NIR spectrophotometer used in this research was constructed by Soma Optics, Ltd. (Tokyo, Japan). An In GaAs linear image sensor with a grating unit (G9211256S; Hamamatsu Co., Ltd., Hamamatsu, Japan) was employed in this spectrophotometer and six halogen lamps (15W; Hamai Electric Lamp Co., Ltd., Tokyo, Japan) with corrective lenses were circularly arranged as an illuminator unit. The illuminated area was approximately $40 \mathrm{~mm}$ in diameter for each lamp. The lineal detector sensor was placed at the center of the illuminator unit, and the distance between the sample surface and the detector head was approximately $70 \mathrm{~mm}$. The spectrophotometer was mounted on a conveyer belt for online simulation of industry conditions. The online NIR spectra measurement was performed in the wavelength range of $872 \mathrm{~nm}$ to $1618 \mathrm{~nm}$ at $1 \mathrm{~nm}$ intervals with a $10 \mathrm{~nm}$ spectral resolution. The exposure time for one scan was fixed at 10 milliseconds and defined based on the need to keep the signal-to-noise $(\mathrm{S} / \mathrm{N})$ ratio below $50 \mu \mathrm{m}$ absorbance. The original software controlling the conveyer belt and NIR spectrophotometer were programmed by Visual Studio (Microsoft, Washington, D.C., United States).
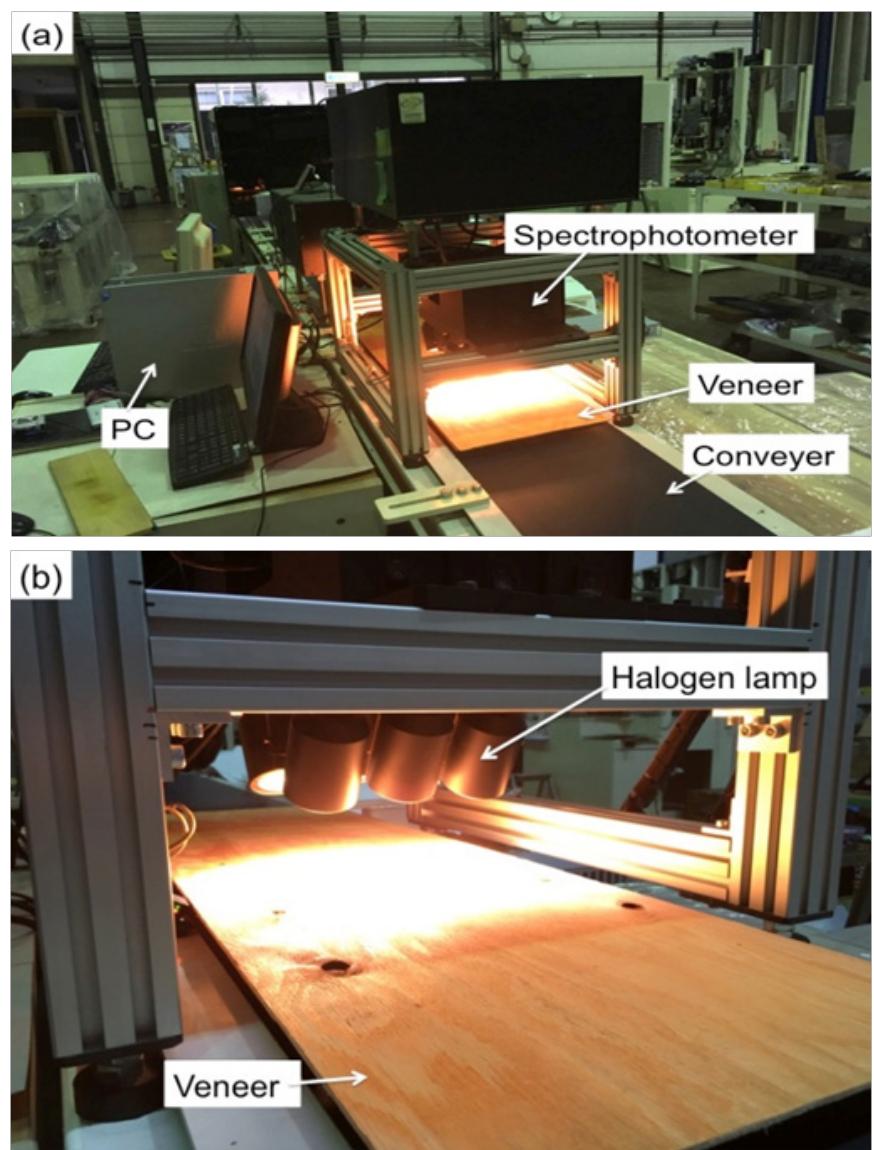

Figure $3(\mathrm{~A})$ and (B) Online NIR spectra measurement.

The online NIR spectra were obtained from tangential surface of the veneer run in the speed of $120 \mathrm{~m} / \mathrm{min}$, regarded as the fastest developed online NIR spectroscopic system for wood industry application together with the same speed as developed by Meder et al. ${ }^{8}$ though they didn't apply the device for veneer samples but for lumber. Two areas were scanned in each veneer sample with 10 scans per area. The results from these two areas were then averaged to obtain a single spectral estimate.

The MOE data from three-point bending test and MC data calculated from oven-dry method served as reference values or measured values. Then these reference values were regressed with their correlated online NIR spectra data to construct the prediction model in NIR data analysis. NIR data analysis is also complicated by the overlapping peaks, apart from chemical information physical properties are also reflected in the spectra, while differences between 
samples result in only small spectral differences. Multivariate data analysis or chemo metrics is used to extract suitable information from the spectra that would correlate with the measured property under investigation; mathematical procedures are used to remove unwanted information without losing important or required information. ${ }^{9}$ In this research, leave-one-out (LOO) and cross-validation partial least square regression (CV-PLSR) were performed.

Wavelengths from 900-1600nm were selected for the next preprocessing spectra treatment data analysis. Many pre-processing treatments on the spectra such as SNV (standard normal variate), MSC (multiplicative scatter correction), 1st derivative with 25 smoothingpoint (SP) (Savitzky-Golay algorithm), 2nd derivative with 25 SP (Savitzky-Golay algorithm), and their combinations were applied. Mean-centering was applied to all spectra while CV-PLSR analysis was being performed. The best pre-processing spectra treatment was determined by the lowest value of the root mean square error for crossvalidation (RMSECV) followed by its coefficient of determination for cross-validation $\left(\mathrm{R}^{2} \mathrm{CV}\right)$ and the optimum number of latent variables (LV) obtained from CV-PLSR analysis. MATLAB R2016b (The Math Works, Inc., Natick, Massachusetts, United States) was used for multivariate data analysis.

\section{Results and discussion}

Table 1 summarizes the statistical data for MOE and MC. Douglasfir veneer has higher mean MOE value of $13.7 \mathrm{GPa}$ than $7.4 \mathrm{GPa}$ of Japanese cedar veneer, with wider sample variance around $16 \mathrm{GPa}$ compared to $9 \mathrm{Gpa}$, respectively. The mean MC for Japanese cedar veneer was $20.0 \%$ and $15.0 \%$ for Douglas-fir veneer. The range of MC for both Japanese cedar veneer and Douglas-fir veneer is around $17-18 \%$.

Table I Statistical data of MOE and MC*

\begin{tabular}{lllllll}
\hline Veneer & Parameter & N & Min & Max & Mean & SD \\
\hline Japanese & MOE (GPa) & 216 & 3.2 & 12.5 & 7.4 & 1.29 \\
cedar & MC (\%) & 68 & 12.4 & 30.1 & 20 & 5.04 \\
\multirow{2}{*}{ Douglas-fir } & MOE (GPa) & 160 & 5.6 & 21.7 & 13.7 & 2.65 \\
& MC (\%) & 138 & 11.9 & 29.6 & 15 & 3.27 \\
\hline
\end{tabular}

*N, Number of sample; SD, standard deviation

The best MOE and MC prediction models for Japanese cedar veneer resulted from the original spectra and the 1st derivative spectra, respectively. While both the MOE and MC prediction models for Douglas-fir veneer resulted from their 1st derivative spectra. Figure 4 shows the best spectra for $\mathrm{MOE}$ and $\mathrm{MC}$ prediction for
Japanese cedar and Douglas-fir veneer. Spectra obtained from MOE measurement show straight softer shape than more bumpy shape on MC measurement's spectra. This straight softer shape of spectra were obtained in the same air-dry MC of the veneers samples, while the spectra of MC measurement were measured in various MC between $12-30 \%$ of the veneer samples. Such differences showed that NIR measurement is clearly affected by moisture content inside the materials measured.
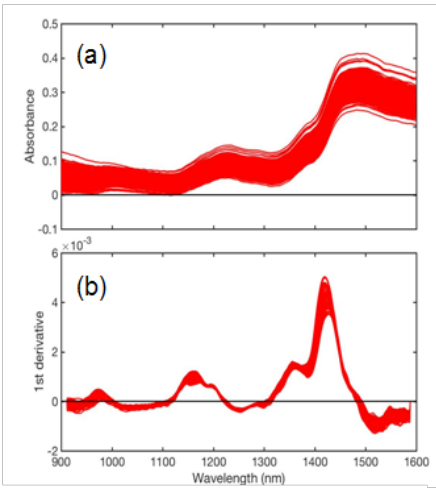
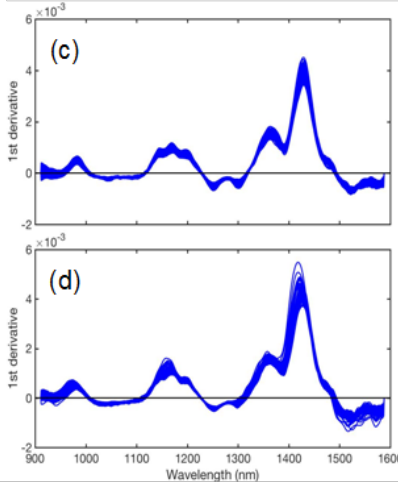

Figure 4 (A) Original spectra for MOE prediction from Japanese cedar veneer (B) Ist derivative spectra for $M C$ prediction from Japanese cedar veneer and (C) Ist derivative spectra for MOE prediction from Douglas-fir veneer (D) Ist derivative spectra for MC prediction from Douglas-fir veneer.

The $120 \mathrm{~m} / \mathrm{min}$ speed of the online NIR spectroscopic system was successfully used to construct the MOE and MC prediction model. Table 2 summarizes the variable data from $\mathrm{MOE}$ and $\mathrm{MC}$ prediction models for Japanese cedar and Douglas-fir veneer from CV-PLSR analysis. The MC prediction model seems to be giving better prediction accuracy than the MOE prediction model. The RMSECV from MOE prediction obtained for Japanese cedar and Douglas-fir veneer are $0.9 \mathrm{GPa}$ and $1.8 \mathrm{GPa}$, respectively, on the LVs 6 and 4 . The $\mathrm{R}^{2} \mathrm{CV}$ from MOE prediction from Japanese cedar and Douglas-fir veneer are 0.43 and 0.52 , respectively. The RMSECV values from MC prediction are $1.0 \%$ for Japanese cedar veneer and $0.6 \%$ for Douglas-fir veneer on LVs 4 and 5, respectively. The $\mathrm{R}^{2} \mathrm{CV}$ values obtained are high with the value of 0.95 for Japanese cedar veneer and 0.96 from Douglas-fir veneer Meder et al. ${ }^{8}$ Also constructed a gantry that enabled NIR instrument to transverse the length of the cant at a speed of $c a .2 \mathrm{~m} / \mathrm{s}$ or the same speed with this research to predict the stiffness of the Pinus radiata cants. When we compared this research to Meder et al. ${ }^{8}$ only focusing on the speed not on the samples being used, the $\mathrm{R}^{2} \mathrm{CV}$ resulted from their research is almost exactly same (0.53) to Douglas-fir veneer's $\mathrm{R}^{2} \mathrm{CV}$, with RMSEP (root mean square error for prediction) value of $1.47 \mathrm{GPa}$ on the optimum number of $\mathrm{LV}$ 12 using 411 samples.

Table 2 Variable data from MOE and MC prediction model for Japanese cedar and Douglas-fir veneer from CV-PLSR analysis*

\begin{tabular}{llllllll}
\hline Veneer & Prediction & LV & $\mathbf{R}^{2} \mathbf{C V}$ & $\mathbf{R}^{2} \mathbf{C}$ & RMSECV & RMSEC & RPD \\
\hline Japanese cedar & MOE (GPa) & 6 & 0.43 & 0.5 & 0.9 & 0.9 & 1.35 \\
& MC (\%) & 4 & 0.95 & 0.97 & I & 0.8 & 4.8 \\
MOE (GPa) & 4 & 0.52 & 0.59 & 1.8 & 1.7 & 1.47 \\
Mouglas-fir & MC (\%) & 5 & 0.96 & 0.97 & 0.6 & 0.5 & 5.15
\end{tabular}

*MOE, modulus of elasticity; MC, moisture content; LV, number of latent variables; R2C, coefficient of determination for calibration; R2CV, coefficient of determination for cross-validation; RMSEC, root mean square error for calibration; RMSECV, root mean square error for cross-validation; RPD, ratio of performance to deviation 
The best RMSECV (lowest) and $\mathrm{R}^{2} \mathrm{CV}$ (highest) values from the $\mathrm{MOE}$ and MC prediction models are also shown on the scatter plots of the measured and predicted of MOE and MC in Figure 5. The online NIR spectra measurement gave lower prediction accuracy compared to the static measurement for veneer stiffness predictions such as done by Fujimoto et al. ${ }^{3}$ Carneiro et al. ${ }^{6}$ Adedipe et al. ${ }^{4}$ The MC prediction in this research gave better prediction accuracy than MOE prediction comparatively.
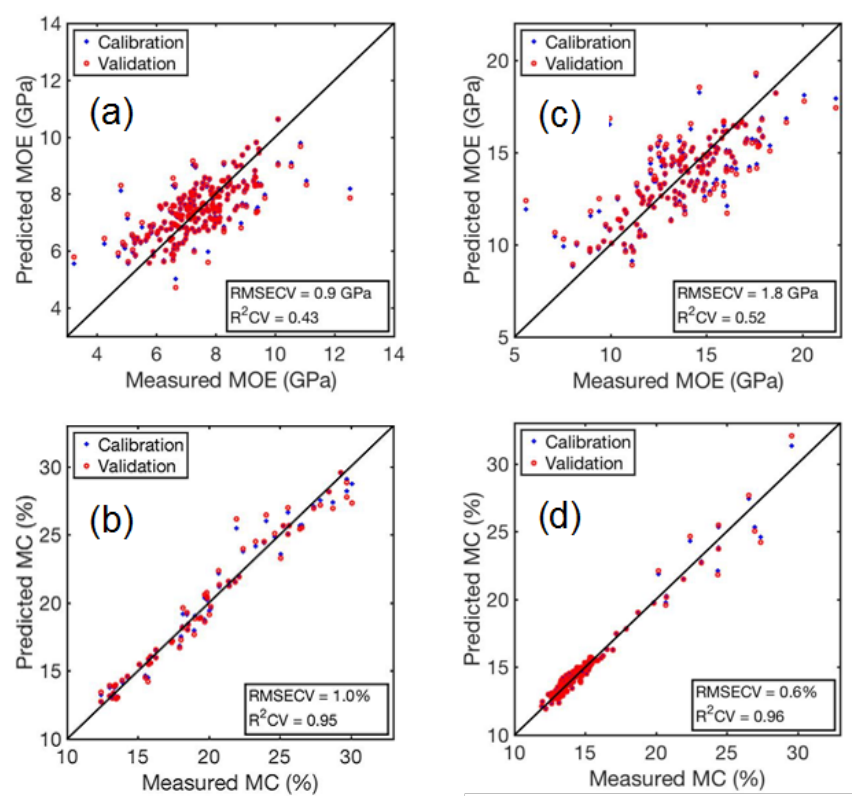

Figure 5 Scatter plots of measured and predicted (A) MOE for Japanese cedar veneer (B) MC for Japanese cedar veneer (C) MOE for Douglas-fir veneer (D) MC for Douglas-fir veneer.

We assure that this speed of NIR spectra acquisition is amazing and compatible for wood industry though the $\mathrm{R}^{2} \mathrm{CV}$ resulted from the very fast speed online system developed like this is still yet acceptable/ reasonable. Yet we need to improve the device to give higher value on the coefficient of determination for validation. Before asserting whether this technology is ready or not yet, we shall do many more tests using different species on various wood products for data collection for evaluation of the online NIR spectroscopic system device.

\section{Conclusion}

This study examined online NIR spectroscopic system as a novel technology for quick assessment for MOE and MC of veneer materials in plywood industry. The fast speed of $120 \mathrm{~m} / \mathrm{min}$ was successfully run to obtain the online NIR spectra of the veneer. Prediction models for both $\mathrm{MOE}$ and $\mathrm{MC}$ for Japanese cedar and Douglas-fir veneer were successful constructed with sufficient prediction accuracy. Even though the fast speed of online NIR spectra acquisition has shown satisfactory results for $\mathrm{MOE}$ and $\mathrm{MC}$ to generate a prediction model for application, more improvements to increase the value of coefficient of determination for validation from this device needs to be done to collect more data for evaluation then convince the wood industry the technology is ready for widespread use.

\section{Acknowledgements}

None.

\section{Conflict of interest}

Authors declare that there is no conflict of interest.

\section{References}

1. Forestry Agency Ministry of Agriculture Forestry and Fisheries of Japan. Annual Report on Forest and Forestry in Japan Fiscal Year 2015. Japan; 2015. p. 1-37.

2. Tsuchikawa S, Kobori H. A review of recent application of near infrared spectroscopy to wood science and technology. Journal of Wood Science. 2015;61(3):213-220.

3. Fujimoto T, Keisuke Kawakami, Haruhisa Aimi, et al. Prediction of dry veneer stiffness using near infrared spectra from transverse section of green log. Journal of Wood Science. 2013;59(5):383-388.

4. Adedipe OE, Dawson-Andoh. Prediction of yellow-poplar (Liriodendron tulipifera) veneer stiffness and bulk density using near infrared spectroscopy and multivariate calibration. Proceedings of the 51st International Convention of Society of Wood Science and Technology. Chile; 2008. 1-9p.

5. Koumbi-Mounanga T, Ung T, Cooper P, et al. Surface quality sensing of trembling aspen (Populus tremuloides Michx) veneer products by near infrared spectroscopy. Wood Materials Science \& Engineering. 2014;10(1).

6. Carneiro ME, Magalhaes WLE, de Muniz GIB, et al. Near-infrared spectroscopy and chemometrics for predicting specific gravity and flexural modulus of elasticity of Pinus spp. veneers. Journal of Near Infrared Spectroscopy. 2010;18(6):481-489.

7. Meder R, Thumm A, Bier H. Veneer stiffness predicted by NIR spectroscopy calibrated using mini-LVL test panels. Holz als Roh-und Werkstoff. 2002;60(3):159-164.

8. Meder R, Thumm A, Marston D. Sawmill trial of at-line prediction of recovered lumber stiffness by NIR spectroscopy of Pinus radiata cants. Journal of Near Infrared Spectroscopy. 2003;11(2):137-143.

9. Manley M. Near-infrared spectroscopy and hyperspectral imaging: nondestructive analysis of biological materials. Chemical Society Reviews. 2014;43:8200-8214. 